\title{
Partonic quasi-distributions of the pion in chiral quark models
}

\section{Wojciech Broniowski*i}

The H. Niewodniczański Institute of Nuclear Physics, Polish Academy of Sciences, 31-342 Cracow, Poland, and Institute of Physics, Jan Kochanowski University, 25-406 Kielce, Poland

E-mail: Wojciech.Broniowskieifj.edu.pl

\section{Enrique Ruiz Arriola}

Departamento de Física Atómica, Molecular y Nuclear and Instituto Carlos I de Fisica Teórica y Computacional, Universidad de Granada, E-18071 Granada, Spain

E-mail: earriola@ugr.es

\begin{abstract}
The evaluation of partonic distributions presents a challenge for QCD, and in particular for its Euclidean lattice realization. Recently, objects called quasi-distributions (which become standard distributions in a limit of the longitudinal momentum of the target hadron going to infinity) have been proposed. We present a non-perturbative, dynamical evaluation of the quark quasidistribution amplitude (QDA) of the pion in the framework of chiral quark models (the NambuJona-Lasinio model and the spectral quark model). We arrive at simple but nontrivial analytic expressions, where the dependence on the longitudinal momentum, the momentum fraction, or the transverse-momentum (for the unintegrated objects) can be explicitly assessed. For the parton distribution amplitude (PDA), we carry out the necessary QCD evolution from the constituent quark model scale to higher scales accessible on the lattice, and compare favorably to the LaMET data.
\end{abstract}

XVII International Conference on Hadron Spectroscopy and Structure - Hadron2017

25-29 September, 2017

University of Salamanca, Salamanca, Spain

\footnotetext{
*Speaker.

${ }^{\dagger}$ Supported by the Polish National Science Center grant 2015/19/B/ST2/00937.

${ }^{\ddagger}$ Supported by the Spanish Mineco grant FIS2014-59386-P, and by the Junta de Andalucía grant FQM225-05.
} 
This talk presents a follow up of [1], where more details of our approach can be found. Techniques used in this work were also recently reported in [2]. The relevant lattice studies of the quasi-distributions in the pion were published in [3]. The cousin problem of the Euclidean lattice extraction of the partonic quasi-distributions [4] in the nucleon attracted a lot of attention from the simulation side [5-8] as well as in theoretical developments [9-25]. A community white paper has also just appeared [26]. For brevity, we present here the distribution amplitudes only, however, the results are analogous for the distribution functions [1].

The definition of the pion quasi-distribution amplitude (QDA) [4] is given by the matrix elements of the bilocal quark operators,

$$
\tilde{\phi}\left(y, P_{3}\right)=\left.\frac{i}{f} \int \frac{d z_{3}}{2 \pi} e^{-i(y-1) z_{3} P_{3}}\left\langle\pi(P)\left|\bar{\psi}(0) \gamma^{3} \gamma_{5} U(0, z) \psi(z)\right| 0\right\rangle\right|_{z_{0}=0, z_{T}=0} .
$$

Similarly, the pion light-cone wave function (LCWF) is

$$
\Psi\left(x, \boldsymbol{k}_{T}\right)=\left.\frac{i}{f} \int \frac{d z_{-}}{2 \pi} e^{i(x-1) z_{-} P_{+}} \int \frac{d^{2} z_{T}}{(2 \pi)^{2}} e^{-i z_{T} \cdot \boldsymbol{k}_{T}}\left\langle\pi(P)\left|\bar{\psi}(0) \gamma^{+} \gamma_{5} U(0, z) \psi(z)\right| 0\right\rangle\right|_{z_{+}=0} .
$$

Above, $z$ denotes the spatial separation of the quark operators (isospin indices are suppressed for brevity), $P^{\mu}$ is the four-momentum of the pion, $y \in(-\infty, \infty)$ is the fraction of $P_{z}$, and $x \in[0,1]$ is the fraction of the light-cone momentum $P_{+}$carried by the valence quark. The gauge link operator $U(0, z)$ is neglected in chiral quark models.

Following the Lorentz covariance of the matrix elements of the quark bilinears, Radyushkin [27] derived an important relation

$$
\tilde{\phi}\left(y, P_{3}\right)=\int_{-\infty}^{\infty} d k_{1} \int_{0}^{1} d x P_{3} \Psi\left(x, k_{1},(x-y) P_{3}\right) .
$$

Thus, QDA can be obtained from LCWF via a straightforward double integration. From rotational symmetry, $\Psi\left(x, \boldsymbol{k}_{T}\right)=\Psi\left(x, k_{T}^{2}\right)$. A similar methodology, also based entirely on the Lorentz covariance, was used in [28-31] to derive the transversity relations between the light-cone and equal-time wave functions (Bethe-Salpeter amplitudes) of the pion.

The standard parton distribution amplitude (PDA) of the pion is obtained as the limit [4] $\phi(x=y)=\lim _{P_{3} \rightarrow \infty} \tilde{\phi}\left(y, P_{3}\right)$, where the support $x \in[0,1]$ is regained. Other pertinent quantities are related to the above definitions via Fourier transforms. The pseudo-distribution amplitude (pseudo-DA) [27] is given by the Fourier transform of the LCWF and noticing that from rotation invariance in depends in general on $|\boldsymbol{z}|$ :

$$
\mathscr{P}(x,|z|)=\int d^{2} k_{T} e^{i z_{T} \cdot k_{T}} \Psi\left(x, \boldsymbol{k}_{T}\right),
$$

where $|z|=z_{T}$. In particular, in the frame $P=\left(E, 0,0, P_{3}\right)$ we may chose $z=\left(0,0,0, z_{3}\right)$, where $x$ is the Fourier conjugate variable of $P \cdot z=-P_{3} z_{3}$. Next, the Ioffe-time distribution amplitude (IDA) $[27,32]$ is simply related to the pseudo-DA:

$$
\mathscr{M}(v,|z|)=\int_{0}^{1} d x e^{i\left(x-\frac{1}{2}\right) v} \mathscr{P}(x,|z|)
$$


Table 1: Analytic formulas for various objects describing the valence quark distribution in the pion, evaluated in the NJL and SQM chiral quark models at the quark model scale and in the chiral limit, $m_{\pi}=0$.

\begin{tabular}{|c|c|c|}
\hline $\begin{array}{l}\text { Name } \\
\text { Symbol }\end{array}$ & NJL & SQM \\
\hline $\begin{array}{l}\mathrm{DA} \\
\phi(x)\end{array}$ & $\theta[x(1-x)]$ & $\theta[x(1-x)]$ \\
\hline $\begin{array}{l}\text { QDA } \\
\tilde{\phi}\left(y, P_{3}\right)\end{array}$ & $\begin{array}{l}\left.\frac{N_{c} M^{2}}{4 \pi^{2} f^{2}} \operatorname{sgn}(y) \ln \frac{P_{3}|y|+\sqrt{M^{2}+P_{3}^{2} y^{2}}}{M}\right|_{\text {reg }} \\
\quad+(y \leftrightarrow 1-y)\end{array}$ & $\begin{aligned} \frac{1}{\pi}[ & \frac{2 m_{\rho} P_{3} y}{m_{\rho}^{2}+4 P_{3}^{2} y^{2}}+\operatorname{arctg}\left(\frac{2 P_{3} y}{m_{\rho}}\right) \\
& +(y \leftrightarrow 1-y)\end{aligned}$ \\
\hline $\begin{array}{l}\text { LCWF } \\
\Psi\left(x, k_{\perp}\right)\end{array}$ & $\left.\frac{N_{c} M^{2}}{4 \pi^{2} f^{2}} \frac{1}{k_{T}^{2}+M^{2}}\right|_{\mathrm{reg}} \theta[x(1-x)]$ & $\frac{6 m_{\rho}^{3}}{\pi\left(4 k_{\perp}^{2}+m_{\rho}^{2}\right)^{5 / 2}} \theta[x(1-x)]$ \\
\hline $\begin{array}{l}\text { pseudo-DA } \\
\mathscr{P}(x,|z|)\end{array}$ & $\left.\frac{N_{c} M^{2}}{4 \pi^{3} f^{2}} K_{0}(M|z|)\right|_{\mathrm{reg}} \theta[x(1-x)]$ & $\frac{1}{2} e^{-\frac{m \rho|z|}{2}}\left(m_{\rho}|z|+2\right) \theta[x(1-x)]$ \\
\hline $\begin{array}{l}\text { IDA } \\
\mathscr{M}(v,|z|)\end{array}$ & $\left.\frac{N_{c} M^{2}}{2 \pi^{3} f^{2}} \frac{\sin \left(\frac{v}{2}\right)}{v} K_{0}(M|z|)\right|_{\mathrm{reg}}$ & $\frac{\sin \left(\frac{v}{2}\right)}{v} e^{-\frac{m_{\rho}|z|}{2}}\left(m_{\rho}|z|+2\right)$ \\
\hline $\begin{array}{l}\text { VDA } \\
\Phi(x, \mu)\end{array}$ & $\left.\frac{N_{c} M^{2}}{4 \pi^{2} f^{2}} \mu e^{-\mu M^{2}}\right|_{\mathrm{reg}} \theta[x(1-x)]$ & $\frac{\mu^{5 / 2} m_{\rho}^{3} e^{-\frac{1}{4} \mu m_{\rho}^{2}}}{4 \sqrt{\pi}} \theta[x(1-x)]$ \\
\hline
\end{tabular}

(we shift $x$ by $\frac{1}{2}$ to get real expressions). Finally, the virtuality distribution amplitude (VDA) [33] is defined via (for simplicity of the resulting expressions we take a real Laplace transform)

$$
\Psi\left(x, k_{\perp}\right)=\frac{1}{\pi} \int_{0}^{\infty} \frac{d \mu}{\mu} e^{-k_{\perp}^{2} \mu} \Phi(x, \mu) .
$$

In chiral quark models, the evaluation of the valence quark distributions in the leading- $N_{c}$ order amounts to computing a one-quark-loop integral (for a review of techniques see, e.g., [34]). For the pion LCWF we get [35]

$$
\Psi\left(x, k_{T}^{2}\right)=\left.\frac{N_{c} M^{2}}{4 \pi^{2} f^{2}} \frac{\theta[x(1-x)]}{k_{T}^{2}+M^{2}-m_{\pi}^{2} x(1-x)}\right|_{\mathrm{reg}} .
$$

The expression in Eq. (7) must be properly regularized (i.e., in a way conserving the proper symmetries). For the NJL model we use the Pauli-Villars regularization (see [36]). The value of the cut-off is chosen in such a way that at a given value of the constituent quark mass $M$ (we use $M=300 \mathrm{MeV}$ ) a proper value for the pion decay constant $f$ follows. In SQM, the regulator is imposed via a spectral integration over the quark mass along a suitably-chosen complex 

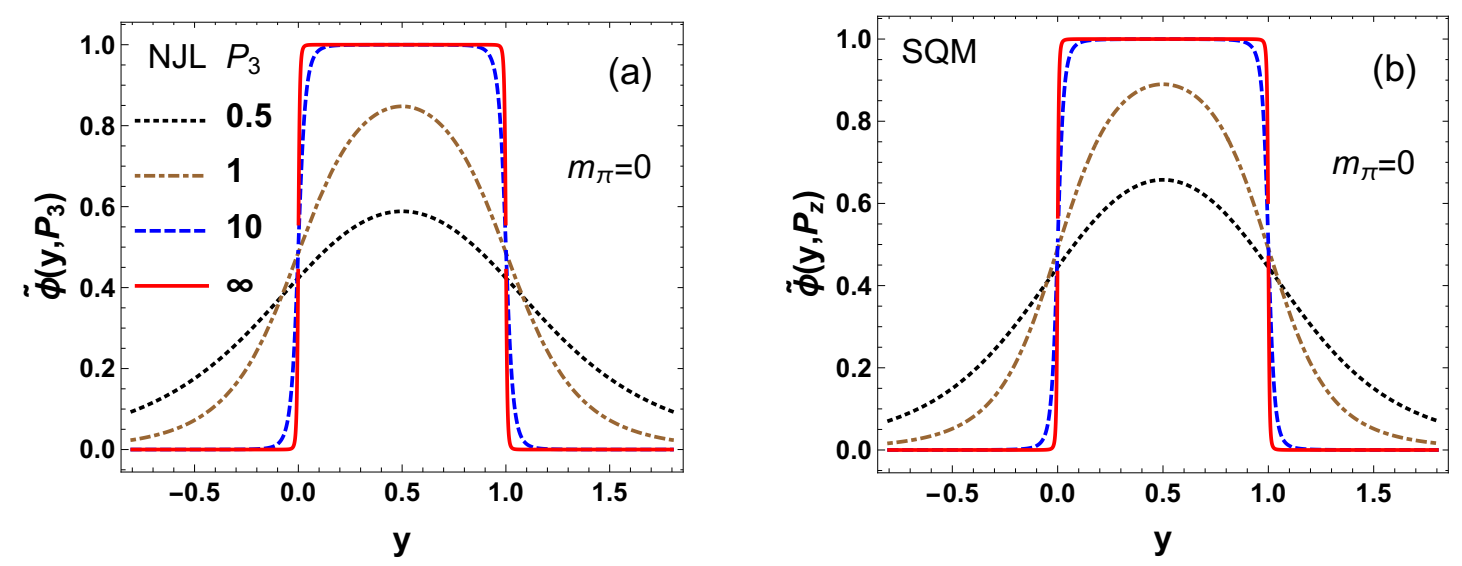

Figure 1: Valence quark quasi-distribution amplitude (QDA) of the pion in (a) NJL and (b) SQM models, obtained at the constituent quark scale $\mu_{0}$ with $m_{\pi}=0$ at various values of the longitudinal momentum $P_{3}$, plotted as a function of the longitudinal momentum fraction $y$.
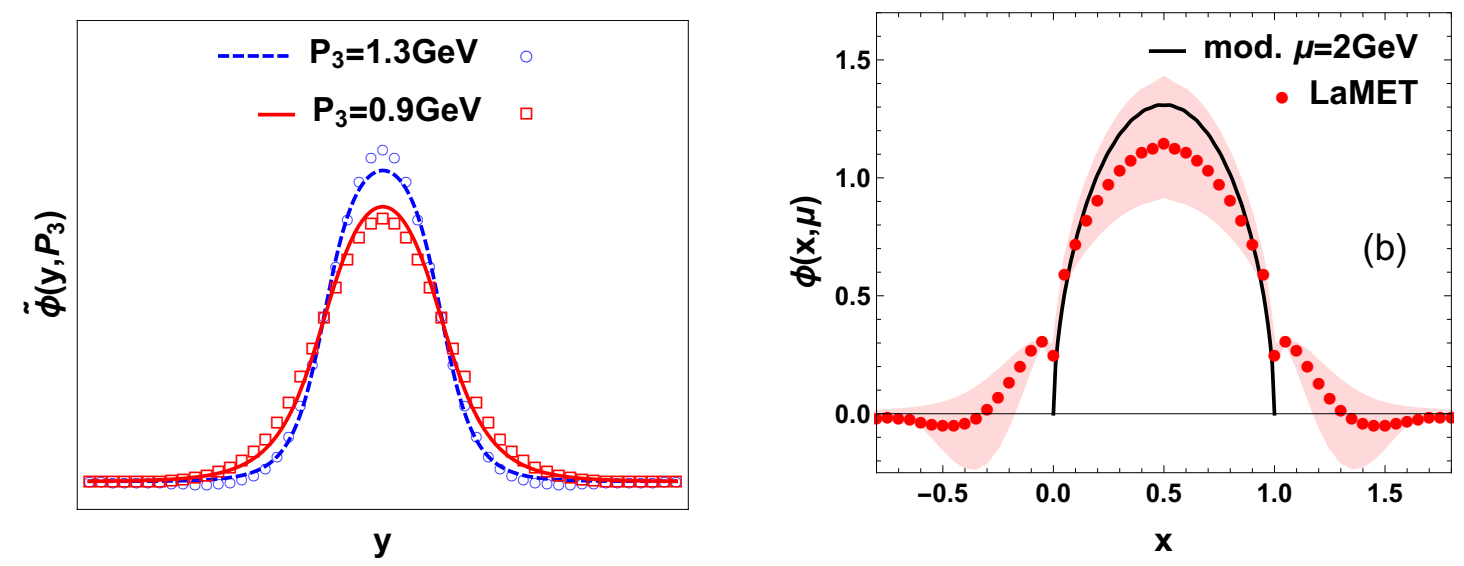

Figure 2: (a) Quark quasi-distribution amplitude (QDA) of the pion in the NJL model at the constituent quark scale $\mu_{0}$, plotted as functions of the longitudinal momentum fraction $y$, evaluated for $P_{3}=0.9$ and $1.3 \mathrm{GeV}$, and compared to the lattice data at $\mu=2 \mathrm{GeV}$ from the LaMET Collaboration at $m_{\pi}=310 \mathrm{MeV}$ [3]. (b) The distribution amplitude (PDA) of the pion, obtained from the NJL model and evolved to $\mu=2 \mathrm{GeV}$, compared to the extraction from the LaMET data [3].

contour [37,38]. The prescription reproduces the phenomenological monopole shape of the pion electromagnetic form factor, $F(t)=1 /\left(1-t / m_{\rho}^{2}\right)$, where $m_{\rho}$ is the mass of the $\rho$ meson.

We note from Eq. (7) that the longitudinal-transverse factorization at the quark model scale $\mu_{0} \simeq 320 \mathrm{MeV}$ [34], i.e., the factorization between the $x$ and $k_{T}$ variables, holds only in the strict chiral limit.

The results for the NJL and SQM models are analytic, but they are particularly simple in the chiral limit of $m_{\pi}=0$. They are collected in Table 1. The QDAs in the chiral limit at various values of $P_{3}$ are plotted in Fig. 1. We note almost identical results from both models. Comparison to the LaMET data [3], very favorable taking into account the simplicity of the model, is made in Fig. 2. 


\section{References}

[1] W. Broniowski and E. Ruiz Arriola, Nonperturbative partonic quasidistributions of the pion from chiral quark models, Phys. Lett. B773 (2017) 385-390, [1707 . 09588].

[2] W. Broniowski and E. Ruiz Arriola, Partonic quasi-distributions of the proton and pion from transverse-momentum distributions, 1711.03377.

[3] J.-H. Zhang, J.-W. Chen, X. Ji, L. Jin and H.-W. Lin, Pion Distribution Amplitude from Lattice QCD, Phys. Rev. D95 (2017) 094514, [1702.00008].

[4] X. Ji, Parton Physics on a Euclidean Lattice, Phys. Rev. Lett. 110 (2013) 262002, [1305. 1539 ].

[5] C. Alexandrou, K. Cichy, V. Drach, E. Garcia-Ramos, K. Hadjiyiannakou, K. Jansen et al., Lattice calculation of parton distributions, Phys. Rev. D92 (2015) 014502, [1504.07455].

[6] C. Alexandrou, K. Cichy, M. Constantinou, K. Hadjiyiannakou, K. Jansen, F. Steffens et al., Updated Lattice Results for Parton Distributions, Phys. Rev. D96 (2017) 014513, [1610 . 03689 ].

[7] C. Alexandrou, K. Cichy, M. Constantinou, K. Hadjiyiannakou, K. Jansen, H. Panagopoulos et al., Progress in computing parton distribution functions from the quasi-PDF approach, in 35th International Symposium on Lattice Field Theory (Lattice 2017) Granada, Spain, June 18-24, 2017, 2017, 1709.07513 , https://inspirehep.net/record/1625112/files/arXiv:1709.07513.pdf.

[8] K. Orginos, A. Radyushkin, J. Karpie and S. Zafeiropoulos, Lattice QCD exploration of pseudo-PDFs, 1706.05373.

[9] X. Xiong, X. Ji, J.-H. Zhang and Y. Zhao, One-loop matching for parton distributions: Nonsinglet case, Phys. Rev. D90 (2014) 014051, [1310 . 7471].

[10] X. Ji, Parton Physics from Large-Momentum Effective Field Theory, Sci. China Phys. Mech. Astron. 57 (2014) 1407-1412, [1404.6680].

[11] Y.-Q. Ma and J.-W. Qiu, Extracting Parton Distribution Functions from Lattice QCD Calculations, 1404.6860 .

[12] X. Ji and J.-H. Zhang, Renormalization of quasiparton distribution, Phys. Rev. D92 (2015) 034006, [1505.07699].

[13] X. Ji, A. Schäfer, X. Xiong and J.-H. Zhang, One-Loop Matching for Generalized Parton Distributions, Phys. Rev. D92 (2015) 014039, [1506.00248].

[14] A. Radyushkin, Nonperturbative Evolution of Parton Quasi-Distributions, Phys. Lett. B767 (2017) 314-320, [1612.05170].

[15] C. Monahan and K. Orginos, Quasi parton distributions and the gradient flow, JHEP 03 (2017) 116, [1612.01584].

[16] J.-W. Chen, X. Ji and J.-H. Zhang, Improved quasi parton distribution through Wilson line renormalization, Nucl. Phys. B915 (2017) 1-9, [1609.08102].

[17] X. Ji, J.-H. Zhang and Y. Zhao, More On Large-Momentum Effective Theory Approach to Parton Physics, 1706.07416.

[18] J.-W. Chen, T. Ishikawa, L. Jin, H.-W. Lin, Y.-B. Yang, J.-H. Zhang et al., Parton Distribution Function with Non-perturbative Renormalization from Lattice QCD, 1706.01295.

[19] C. E. Carlson and M. Freid, Lattice corrections to the quark quasidistribution at one-loop, Phys. Rev. D95 (2017) 094504, [1 702 . 05775]. 
[20] R. A. Briceno, M. T. Hansen and C. J. Monahan, Role of the Euclidean signature in lattice calculations of quasidistributions and other nonlocal matrix elements, Phys. Rev. D96 (2017) 014502 , [1703.06072].

[21] G. C. Rossi and M. Testa, Note on lattice regularization and equal-time correlators for parton distribution functions, Phys. Rev. D96 (2017) 014507, [1706. 04428 ].

[22] I. W. Stewart and Y. Zhao, Matching the Quasi Parton Distribution in a Momentum Subtraction Scheme, 1709.04933.

[23] J.-W. Chen, T. Ishikawa, L. Jin, H.-W. Lin, A. Schäfer, Y.-B. Yang et al., Gaussian-weighted Parton Quasi-distribution, 1711.07858.

[24] W. Wang, S. Zhao and R. Zhu, A Complete Matching for Quasi Parton Distribution Functions at One-Loop Order, 1708.02458.

[25] T. J. Hobbs, Quantifying finite-momentum effects in meson quasi-PDFs, 1708.05463.

[26] H.-W. Lin et al., Parton distributions and lattice QCD calculations: a community white paper, 1711.07916.

[27] A. V. Radyushkin, Quasi-PDFs, momentum distributions and pseudo-PDFs, 1705.01488.

[28] G. A. Miller and B. C. Tiburzi, The Relation Between Equal-Time and Light-Front Wave Functions, Phys. Rev. C81 (2010) 035201, [0911.3691].

[29] W. Broniowski, S. Prelovsek, L. Santelj and E. Ruiz Arriola, Pion wave function from lattice QCD vs. chiral quark models, Phys. Lett. B686 (2010) 313-318, [0 911 . 4705].

[30] E. Ruiz Arriola and W. Broniowski, Transversity relations, chiral and holographic models, and pion wave functions from lattice QCD, PoS LC2010 (2010) 041, [1 009 . 5781].

[31] G. A. Miller, Transverse Charge Densities, Ann. Rev. Nucl. Part. Sci. 60 (2010) 1-25, [1 002.0355$].$

[32] V. Braun, P. Gornicki and L. Mankiewicz, Ioffe - time distributions instead of parton momentum distributions in description of deep inelastic scattering, Phys. Rev. D51 (1995) 6036-6051, [hep-ph/9410318].

[33] A. V. Radyushkin, Virtuality Distributions in Application to $\gamma \gamma^{*} \rightarrow \pi^{0}$ Transition Form Factor at Handbag Level, Phys. Lett. B735 (2014) 417-425, [1404 . 7032].

[34] W. Broniowski, E. Ruiz Arriola and K. Golec-Biernat, Generalized parton distributions of the pion in chiral quark models and their QCD evolution, Phys.Rev. D77 (2008) 034023, [0 712 . 1012 ].

[35] E. Ruiz Arriola and W. Broniowski, Pion light-cone wave function and pion distribution amplitude in the Nambu-Jona-Lasinio model, Phys. Rev. D66 (2002) 094016, [hep-ph / 020726 6].

[36] C. Schuren, E. Ruiz Arriola and K. Goeke, Explicit chiral symmetry breaking in the Nambu-Jona-Lasinio model, Nucl. Phys. A547 (1992) 612-632.

[37] E. Ruiz Arriola, Parton distributions for the pion in a chiral quark model, in Proceedings, Workshop on Lepton Scattering, Hadrons and QCD: Adelaide, Australia, March 26-April 6, 2001, pp. 37-44, 2001, hep-ph/0107087, DOI.

[38] E. Ruiz Arriola and W. Broniowski, Spectral quark model and low-energy hadron phenomenology, Phys. Rev. D67 (2003) 074021, [hep-ph/0301202]. 